\title{
Desafios e propostas para ampliação da testagem e diagnóstico para COVID-19 no Brasil
}

\author{
Challenges and proposals for scaling up COVID-19 testing and \\ diagnosis in Brazil
}

\author{
Laio Magno (https://orcid.org/0000-0003-3752-0782) ${ }^{1}$ \\ Thais Aranha Rossi (https://orcid.org/0000-0002-2561-088X) ${ }^{1}$ \\ Fernanda Washington de Mendonça-Lima (https://orcid.org/0000-0003-4444-5805) ${ }^{2}$ \\ Carina Carvalho dos Santos (https://orcid.org/0000-0002-2898-0851) ${ }^{2}$ \\ Guilherme Barreto Campos (https://orcid.org/0000-0002-0089-9517) ${ }^{3}$ \\ Lucas Miranda Marques (https://orcid.org/0000-0002-8276-8149) ${ }^{3}$ \\ Marcos Pereira (https://orcid.org/0000-0003-3766-2502) ${ }^{4}$ \\ Nilia Maria de Brito Lima Prado (https://orcid.org/0000-0001-8243-5662) ${ }^{3}$ \\ Inês Dourado (https://orcid.org/0000-0003-1675-2146) ${ }^{4}$
}

\footnotetext{
${ }^{1}$ Departamento de Ciências da Vida, Universidade do Estado da Bahia. R. Silveira Martins 2555, Cabula. 41150-000 Salvador BA Brasil.

laiomagnoss@gmail.com 2 Departamento de Análises Clínicas e Toxicológicas, Faculdade de Farmácia, Universidade Federal da Bahia (UFBA). Salvador BA Brasil.

${ }^{3}$ Instituto Multidisciplinar em Saúde, UFBA. Vitória da Conquista BA Brasil

${ }^{4}$ Instituto de Saúde

Coletiva, UFBA. Salvador BA Brasil
}

\begin{abstract}
The Brazilian context of social inequalities and barriers in accessing health services may deteriorate the situation of the COVID-19 pandemic, which already affects all Brazilian federative states, with the growing curve of increasing confirmed cases and deaths. National governments and scientific field agents have been looking for evidence for the best practices of prevention and control of transmission, and care of infection and disease, including diagnosis, treatment, and health care measures. The large-scale testing strategy, aimed at early diagnosis, quarantine of the mild cases identified, as well as those of the contacts, and adequate care of severe cases, has been revised and indicated as one of the efficient pandemic control measures in several countries in the world. This paper aims to discuss the challenges of COVID-19 testing and diagnosis in Brazil.
\end{abstract}

Key words COVID-19, Testing, Diagnosis, Epidemiological surveillance, Brazil
Resumo O contexto brasileiro de desigualdades sociais e barreiras no acesso aos serviços de saúde pode agravar a situação da pandemia de COVID-19, que já afeta todos os estados da federação, com a curva crescente de aumento de casos confirmados e mortes. O governo dos países e os agentes do campo científico têm buscado evidências para as melhores práticas de prevenção e controle da transmissão, e cuidado da infecção e doença, incluindo medidas de diagnóstico, tratamento e de atenção à saúde. A estratégia de testagem em larga escala, visando o diagnóstico precoce, quarentena dos casos leves identificados, bem como dos contactantes, e cuidado adequado dos casos graves, tem sido revisada e indicada como uma das medidas eficientes para o controle da pandemia em vários países do mundo. O artigo tem como objetivo discutir os desafios da testagem e do diagnóstico de COVID-19 no Brasil.

Palavras-chave COVID-19, Testagem, Diagnóstico, Vigilância epidemiológica, Brasil 


\section{Introdução}

Os coronavírus fazem parte de uma família de vírus que causam infecções respiratórias em humanos, sendo a segunda principal causa do resfriado comum e, até as últimas décadas, quase nunca causavam doenças mais graves. Há sete coronavírus humanos ( $\mathrm{HCoVs}$ ) conhecidos, entre eles o SARS-COV (que causa Síndrome Respiratória Aguda Grave-SRAG), o MERS-COV (síndrome respiratória do Oriente Médio) e o SARS-CoV-2.

Em 31 de dezembro de 2019, o escritório da Organização Mundial de Saúde (OMS) na China recebeu notificações sobre a ocorrência de casos de pneumonia de causa desconhecida entre trabalhadores de um mercado de frutos do mar em Wuhan/China. Em março de 2020, a OMS caracterizou o estado de pandemia dessa doença, denominada de Coronavirus Disease 2019 (COVID-19) ${ }^{1,2}$. O agente etiológico desta doença é o coronavírus subtipo 2, ou SARS-CoV-2 ${ }^{2}$. Desde então, o governo dos países e os agentes do campo científico buscam evidências científicas para as melhores práticas de prevenção e controle da transmissão, e cuidado da infecção e doença incluindo medidas de diagnóstico, tratamento e de atenção à saúde. Até o dia 31 de maio de 2020, já foram confirmados no mundo 5.934 .936 de casos de COVID-19 e 367.166 mortes associadas, o que corresponde a uma taxa de letalidade de $6,2 \%{ }^{3}$. A OMS declarou essa pandemia como uma "emergência de saúde pública de importância internacional".

O SARS-CoV-2 rapidamente se disseminou em várias partes do mundo, indicando alta taxa de transmissibilidade ${ }^{4,5}$. Ainda não existem estudos suficientes relacionando sua alta taxa de transmissão com questões socioeconômicas, mas, notadamente, localidades com baixo índice de desenvolvimento humano (IDH) e alta desigualdade socioeconômica provavelmente possuem alto risco de disseminação do vírus e de registrar maiores taxas de incidência e letalidade pela COVID-19, uma vez que essas comunidades podem apresentar dificuldade em dispor de condições materiais para isolamento social ${ }^{6}$. Outro fator importante é que essas localidades possuem alta densidade demográfica com pessoas vivendo aglomeradas em casas com poucos cômodos, o que dificulta o distanciamento social, considerado fator de risco para transmissão de doenças respiratórias? ${ }^{7}$. Além disso, as populações que moram nestes espaços já sofrem com uma carga maior de outras doenças infecciosas agudas e/ ou doenças crônicas não transmissíveis (DCNT), potenciais fatores de gravidade da COVID-19, tais como infecção pelo HIV e tuberculose ${ }^{8}$, hipertensão e diabetes ${ }^{9,10} \mathrm{e}$ obesidade ${ }^{11}$. Não menos importante ao agravamento da situação nestas comunidades, é a dificuldade de acesso rápido a serviços de saúde e a informações de qualidade que possibilitem o autocuidado ${ }^{6}$.

O Brasil é um dos países que registra uma das mais altas taxas de desigualdade social, com índice de Gini de 53.9 $9^{12}$, ocupando o $2^{\circ}$ lugar em má distribuição de renda no mundo ${ }^{13}$, e apresentando desigualdades regionais profundas. Cerca de $40 \%$ da população brasileira está em situação de trabalho informal ${ }^{14}$, sem acesso a direitos trabalhistas, o que pode dificultar a adesão às medidas de distanciamento social indicadas em decorrência da necessidade de circulação nas ruas devido à manutenção do trabalho e, consequentemente, da renda necessária para sobrevivência. Além disso, o Brasil convive com uma transição epidemiológica incompleta, quando comparamos com países da Europa, por exemplo, o Brasil registra altas taxas de incidência e prevalência de doenças infectocontagiosas e, ao mesmo tempo, de $\mathrm{DCNT}^{15}$. E é neste contexto que a pandemia de COVID-19 chega ao país, em 26 de fevereiro de 2020, com o primeiro caso registrado em São Paulo/SP. Até o dia 31 de maio de 2020 foram registrados 514.849 casos confirmados de COVID-19, e 29.314 óbitos, o que representa uma taxa de letalidade de 5,7\%. As regiões com maior proporção de casos são: Sudeste com 36,4\% $(187.240)$ e Nordeste com $34,8 \%(179.401)^{16}$. Em comparação com a pandemia pelo vírus influenza A (H1N1) pdm09, a pandemia de COVID-19 já ultrapassou os números no Brasil. Entre 20092010, por exemplo, foram registrados um total de 53.797 casos, e uma taxa de letalidade de 3,9\% em $2009^{17}$.

Nos primeiros meses de 2020 no Brasil, houve um aumento expressivo da taxa de incidência da SRAG, a manifestação mais grave da COVID-19, especialmente entre as pessoas com mais de 60 anos, quando comparado com os dados de incidência dos 10 anos anteriores ${ }^{18,19}$. Entretanto, especula-se que essa maior incidência seja devido à subnotificação de casos no Brasil ${ }^{20}$. Estudo registrou grande desigualdade nas taxas de subnotificação de COVID-19 nos diversos estados da federação, com os sete primeiros lugares ocupados por estados da região norte e nordeste ${ }^{21}$. Estudos relatam que os países que não estão testando o suficiente suas populações, apresentam um cenário não confiável da taxa de incidência da infecção ${ }^{22,23}$. Nesse sentido, este artigo tem como obje- 
tivo discutir os desafios da testagem e diagnóstico de COVID-19 no Brasil.

\section{Desafios para o diagnóstico de COVID-19}

O diagnóstico da COVID-19 é um desafio em todo mundo. Entre os aspectos que dificultam: 1) o material biológico a ser utilizado, como por exemplo, swab de naso e/ou orofaringe, plasma, soro ou sangue total; 2) a definição do marcador biológico com maiores chances de ser detectado; 3) o tipo de metodologia empregada (métodos virológicos, biologia molecular e imunoensaios); 4) o momento ideal da infecção para a coleta da amostra e tipo ideal de amostra; e 5) a acurácia dos testes de diagnóstico disponíveis.

Além disso, com o estado de pandemia declarado pela OMS, há dificuldade de obtenção de insumos para maior disponibilização do teste molecular de detecção do RNA viral, uma vez que este se tornou uma necessidade global. $\mathrm{O}$ Brasil depende de importações de muitos materiais, devido ao número limitado de empresas nacionais produtoras ${ }^{24,25}$.

Deve-se considerar que, por mais precisos e rápidos que sejam os métodos de laboratório, o diagnóstico da COVID-19 demanda uma coleta adequada da amostra do paciente no momento certo da infecção, no sentido de aumentar a chance de detecção do marcador biológico investiga$\mathrm{do}^{26}$. O teste confirmatório é a detecção do material genético do vírus, como o RNA viral, por PCR em tempo real (RT-qPCR). Este pode ser detectado em amostras de fezes, urina e sangue, embora com menor sensibilidade e especificidade do que nas amostras respiratórias ${ }^{26}$. Uma exceção é o RNA do SARS-CoV-2 que é constantemente detectado nas fezes até duas semanas após o início dos sintomas ${ }^{27}$. Desse modo, o RT-qPCR do swab combinado oral/nasal é considerado como teste confirmatório para SARS-CoV-2, até o momento. No entanto, existem alguns aspectos limitantes desse teste, como: 1 ) a positividade do teste geralmente ocorre nos primeiros 4 a 8 dias após o aparecimento dos sintomas, geralmente tornando-se negativo após cerca de 14 dias $^{28,29}$; 2) Trata-se de teste de alta complexidade técnica, que necessita de uma infraestrutura com um nível de biossegurança adequado para realização, e é relativamente caro, custando entre $\mathrm{R} \$ 150,00$ a $\mathrm{R} \$ 350,00$ por amostra, com variações significativas entre as instituições fornecedoras ${ }^{26,30}$.

Alguns estudos têm mostrado a importância dos métodos baseados em pesquisa de anticorpos no sangue para o diagnóstico da COVID-19 em indivíduos assintomáticos e sintomáticos, além da produção de dados sobre a resposta imune humoral para o desenvolvimento de vacinas e/ ou tratamento ${ }^{28}$. São chamados de testes de ensaio imunoenzimático (ELISA), e permitem a detecção de anticorpos específicos. Além disso, apresentam vantagem na rapidez dos resultados e detecção, de relativo baixo custo, mas podem apresentar baixa sensibilidade ${ }^{26}$. A duração média da detecção de anticorpos IgM e IgA para infecção pelo SARS-CoV-2 é de 5 dias (intervalo interquartil - IIQ 3-6 dias), enquanto a IgG pode ser detectada em menos de 14 dias (IIQ 10-18 dias) após o início dos sintomas, com uma taxa de positividade de $85,4 \%, 92,7 \%$ e $77,9 \%$, respectivamente $^{31}$. Por outro lado, uma desvantagem relevante dos métodos baseados na detecção de anticorpos, é a possibilidade de reações cruzadas com outros vírus, principalmente os da mesma família, que causam resfriados e outras doenças respiratórias ${ }^{32}$.

Considerando potencialidades e limitações dos principais métodos diagnósticos usados, é importante frisar que as vantagens dos testes baseados em imunoensaios são: 1) o tempo menor de realização e leitura de resultados (testes rápidos variam de 15 a 30 minutos; ELISA de 1 a 2 horas); 2) os testes rápidos (imunocromatografia) podem ser realizados em pesquisa de campo, a partir de gota de sangue colhida na polpa digital; 3) o ELISA, embora de média complexidade, pode ser realizado por automação com resultados obtidos em 2 a 3 horas, no máximo; 3 ) uma vez que se trata de uma infecção aguda, há chance de após 10 a 14 dias, o teste baseado na detecção de RNA viral, não ser positivo, enquanto que os anticorpos da classe IgG poderão ser detectados, em princípio, por toda a vida, além disso os títulos de IgG poderão ser aferidos para demonstrar uma conversão sorológica recente ${ }^{31}$. Embora as informações em relação aos pontos de corte de dias de sensibilidade e especificidade do RT-qPCR e testes imunológicos ainda estejam sob investigação, alguns estudos apontam que quanto mais no início dos sintomas, maior chance de positividade do RT-qPCR, e quanto mais distante do início dos sintomas, maior chance de positividade de IgM e IgG. Os testes com princípios metodológicos de biologia molecular e de imunologia são fundamentais em diferentes momentos da infecção, tendo sido demonstrada a aplicação simultânea com maior proficiência diagnóstica e prognóstica ${ }^{33}$. Desse modo, detectar a produção de anticorpos, especialmente IgM, que são produzidos rapidamente após a infecção, 
pode ser uma ferramenta combinada com a RTqPCR para melhorar a sensibilidade e precisão diagnóstica ${ }^{31}$

\section{Testagem para COVID-19: evidências, estratégias, desafios}

A transmissão do coronavírus pode ocorrer por gotículas de saliva, espirro, tosse ou catarro, que podem ser repassados por toque ou aperto de mão, objetos ou superfícies contaminadas pelo infectado ${ }^{5,34}$, e o diagnóstico precoce de novos casos de COVID-19 através de testagem é crucial para interromper a disseminação do vírus $^{35}$ por meio de estratégias de isolamento social e quarentena.

A confirmação de casos de COVID-19 é um desafio, pois geralmente há um descompasso entre o início da sintomatologia e o diagnóstico laboratorial preciso ${ }^{36}$. Além disso, cerca de $80 \%$ dos casos de COVID-19 são assintomáticos ou oligossintomáticos $^{37}$, e, na maioria das vezes, não são diagnosticados. Na China, ainda no início do surto em Wuhan, estima-se que as infecções não registradas foram a fonte de infecção para 79\% dos casos identificados ${ }^{34}$.

Estudos com doenças infecciosas como tuberculose $^{38} \mathrm{e}$ febre hemorrágica viral africana ${ }^{39}$, além da COVID-1940, destacam como o atraso no diagnóstico e tratamento de indivíduos infectados, são determinantes para a velocidade de disseminação das infecções em contextos de epidemia. Em diversos países, uma das medidas mais efetivas em termos de deteç̧ão e prevenção de novos casos de COVID-19 foi a testagem em ampla escala da população $0^{41-43}$, já recomendada pela $\mathrm{OMS}^{1}$; assim como a indicação do regime de quarentena por 14 dias para os casos identificados, e seus contatos diretos após o início dos sintomas ${ }^{44}$.

Alguns países da Ásia e da Oceania apresentaram controle da pandemia com a combinação de estratégias com a ampla testagem. $\mathrm{Na}$ China, por exemplo, algumas medidas foram importantes para o controle da COVID-19, como a detecção precoce de casos através de testagem, isolamento dos casos e rastreio de todos os contactantes, e atendimento clínico de qualidade para os infecta$\operatorname{dos}^{40}$. Em Singapura, a vigilância epidemiológica incluiu a testagem de todos casos suspeitos e dos contactantes. Desse modo, a estratégia contribuiu para a detecção precoce de aproximadamente metade (53\%) dos casos de COVID-19, além de ter sido uma estratégia efetiva para a diminuição progressiva do aparecimento de casos autóctones da doença logo no início da epidemia ${ }^{41}$. A resposta de Singapura e de Hong-Kong, aponta para a importância da ampliação da testagem, do sistema de vigilância com o controle seletivo dos viajantes, bem como das medidas de financiamento para custeio pelo Estado do tratamento, planejamento, articulação e gerenciamento dos serviços de saúde ${ }^{45}$. A Nova Zelândia conseguiu controlar a pandemia no seu território e eliminar a transmissão comunitária ${ }^{46}$, ao adotar entre outras estratégias (como o lockdown, controle de fronteiras, promoção de educação em saúde etc.), a testagem em ampla escala da população para rastreamento e detecção rápida de casos e contactantes, e implementação de quarenten $a^{47}$. $\mathrm{Na}$ Austrália, as ações de testagem associadas à organização do sistema de saúde aliada às ações de telessaúde, vem ocasionando baixa taxa de letalidade com 7.185 casos confirmados, 103 mortes, e letalidade de $1,4 \%$ em 31 de maio de $2020^{3,48}$.

$\mathrm{Na}$ Europa observamos alguns países que vivenciaram um colapso da rede de atenção hospitalar, como a Itália, que implementou a ampla testagem, inclusive de pessoas assintomáticas, somente após a situação dramática de falta de leitos de terapia intensiva ${ }^{49}$. A Espanha seguiu protocolo de teste para pessoas com sintomas de síndrome respiratória aguda e profissionais de saúde sintomáticos e também excedeu a capacidade instalada de número de leitos de terapia intensiva ${ }^{50}$. O Reino Unido também adotou medidas tardias para enfrentamento da pandemia e de testagem ${ }^{51}$, mas adotou recentemente um protocolo de vigilância que envolve pessoas com sintomatologia semelhantes à influenza, bem como a coleta de amostra sorológica de todas as faixas etárias, e os dados são monitorados periodicamente $^{52}$. Por outro lado, a Alemanha é uma das exceções do continente, mostrando uma reposta rápida e coordenada à pandemia, associada à testagem de forma descentralizada, incluindo desde o início os jovens e os casos com sintomas leves ${ }^{53}$.

Nas Américas, dois países se desatacam, os Estados Unidos da América, maior país da América do Norte, que mostrou uma resposta nacional tardia e não coordenada de testagem e isolamento social ${ }^{50}$, o que pode ter levado à primeira posição no número de casos confirmados de COVID-19 (1.716.078) e mortes (101.567) até 31 de maio de $2020^{54}$. E o Brasil, maior país da América Latina, que registra tendência crescente do número de casos confirmados e óbitos, contando com uma resposta pública federal descoordenad $a^{55} \mathrm{e}$ com limitada testagem da população $0^{21}$, mas que por outro lado possui o Sistema Único de Saúde (SUS) de acesso universal ${ }^{56}$, com um 
amplo Sistema Nacional de Vigilância em Saúde (SNVS), o que favorece a adoção de respostas rápidas de muitos governos regionais e locais.

Desse modo, apesar de não esgotar a literatura sobre a resposta de testagem dos países, ressalta-se a importância das estratégias de testagem ${ }^{42}$ associadas à organização do sistema de saúde no enfrentamento à pandemia, pois o número de casos confirmados permite o monitoramento da progressão da doença $a^{57}$, o que pode evitar o colapso da rede de atenção hospitalar, a partir da articulação entre distintos níveis de atenção à saúde. Além disso, apesar de não conseguirmos ainda isolar o efeito da estratégia de testagem sobre as taxas de incidência da doença, as experiências internacionais registram a importância destes esforços, em articulação com os sistemas de saúde, no controle da pandemia de COVID-19.

\section{Vigilância epidemiológica}

O processo de investigação, notificação e monitoramento de casos de COVID-19 pelo SNVS do SUS, é fundamental na resposta ao COVID-19, pois possui atuação em todo o território brasileiro de modo articulado e hierarquizado, por meio dos Centros de Informações Estratégicas e Respostas em Vigilância em Saúde (CIEVS), das secretariais municipais e estaduais de saúde ${ }^{58}$. Além das atividades rotineiras, o SNVS é acionado na ocorrência de situações de Emergências em Saúde Pública de Importância Nacional, como o caso da pandemia de COVID-1944. Nestes casos, a rede de serviços deve se organizar para dar respostas rápidas com o emprego urgente de medidas de prevenção, controle e contenção de riscos, danos e agravos à saúde pública ${ }^{59}$.

A descentralização da testagem é uma estratégia fundamental para o aumento de detecção de novos casos, vinculação ao cuidado adequado e vigilância epidemiológica, mas enfrenta alguns desafios como as desigualdades socioeconômicas, e na distribuição de equipamentos e infraestrutura disponível para o diagnóstico. Nesse sentido, algumas iniciativas que visam a ampliação de testagem têm sido adotadas pela Agência Nacional de Vigilância Sanitária (ANVISA), como por exemplo a aprovação da aplicação de testes rápidos (TR) em farmácias ${ }^{60}$, e de novos testes diagnósticos para a detecção de anticorpos para SARS-CoV-2 ${ }^{61,62}$. Além disso, uma estratégia nacional de vigilância epidemiológica e laboratorial para a COVID-19 foi lançada, no dia 6 de maio de 2020 , com o objetivo de testar cerca de $22 \%$ da população brasileira ${ }^{63}$.
Entretanto, a estratégia da testagem deve considerar a acurácia dos testes para detecção de anticorpos, pois a sensibilidade e a especificidade dos testes aprovados no Brasil variam entre os kits dos diferentes fabricantes ${ }^{64}$. E há poucos estudos de validação publicados ${ }^{65}$. Por exemplo, dentre os testes aprovados no Brasil a sensibilidade encontra-se em níveis de baixo a moderado, o que pode implicar na dificuldade de detecção de indivíduos infectados, especialmente nos testes para detecção de anticorpos anti-SARS-CoV-2 da classe IgM na fase inicial da infecção ${ }^{65}$. Além disso, o valor preditivo positivo pode apresentar variações com a prevalência da infecção na população, com valores maiores nas populações com prevalência igual ou maior que $10 \%{ }^{66}$. É importante ressaltar também que muitos dos testes disponíveis ainda estão em fase de aperfeiçoamento técnico, além de existirem poucos estudos relacionados à dinâmica dos biomarcadores na resposta imune humoral.

Desse modo, a informação e a comunicação sobre os aspectos limitantes destes testes e a interpretação dos testes pelos profissionais que aplicarão os TR e outros testes são imprescindíveis, para que não haja baixa percepção de risco de transmissão por parte da população, devido, por exemplo, a resultados falsos negativos, pois poderia potencializar a interação social e aumentar a transmissão. Além disso, deve-se considerar que a testagem precisa estar vinculada à notificação e ao acompanhamento do SNVS, bem como à assistência de profissionais de saúde treinados para o cuidado clínico-individual no manejo dos casos, e orientação das limitações de sensibilidade e especificidade dos testes. Faz-se necessário também a garantia de oferta de testagem aos cidadãos brasileiros de comunidades populares que não consigam efetuar compra, bem como do sigilo e confidencialidade dos resultados individuais.

A resposta brasileira e internacional à epidemia de HIV/AIDS demonstrou que a descentralização de testagem é fundamental para o aumento do diagnóstico precoce da infecção, e da rápida vinculação ao cuidado, inclusive por meio da aplicação de TR para triagem por pessoas leigas treinadas ${ }^{67-69}$. Desse modo, a atenção primária à saúde (APS), que é a porta de entrada preferencial no SUS, pode ter um papel importante na ampliação da testagem, pois está presente nos mais distantes locais do território brasileiro ${ }^{70}$, com equipes de agentes comunitários com entrada nas comunidades ${ }^{71}$, e profissionais de saúde treinados na aplicação de outros TR (HIV, sífilis 
e hepatites virais $)^{72}$. Assim, a APS pode se tornar o locus central da descentralização e democratização dos TR para identificação de casos de COVID-19 no território brasileiro, com a realização de busca ativa de casos e contactantes, com a vinculação dos usuários às estratégias de quarentena, e a prestação de cuidado básico aos infectados com sintomatologia leve e a articulação com outros pontos da rede de atenção à saúde.

Nesse sentido, a resposta de testagem populacional para COVID-19 pode ser coordenada pelo SNVS em duas frentes: 1) individual: com o acesso ao teste pela rede de saúde suplementar (serviços privados: hospitais, ambulatórios ou farmácias), com a notificação de casos e o monitoramento pela vigilância epidemiológica local, e cuidado clínico com orientações adequadas; e 2) coletivo: com acesso prioritário pela APS (sem a eliminação do acesso por outros níveis de atenção), efetuando-se a devida notificação de casos, organizado e monitorado através da vigilância epidemiológica local. Além disso, a busca ativa deve ser adotada como ponto de partida de investigação de todos os contactantes dos casos de COVID-19 já confirmados, a partir de teste RTqPCR com amostra de swab oral/nasal coletada em até 7 dias após o início dos sintomas ou imunológico (teste rápido por imunocromatografia ou sorologia por ELISA) após 7 dias do início dos sintomas. Para os assintomáticos, a coleta pode ser baseada na quantidade de dias referida após o contato com a pessoa infectada: $<7$ dias (RTqPCR) e $\geq 7$ dias (imunológico). Os casos confirmados com resultado positivo devem ser testados e recomendados a entrar em quarentena por 14 dias, conforme protocolo do Ministério da Saúde (Figura 1) ${ }^{44}$.

\section{Conclusões}

A ampliação da testagem e do diagnóstico da COVID-19 é um desafio que se impõe à sociedade brasileira e ao SUS. Embora enfrentemos, há anos,

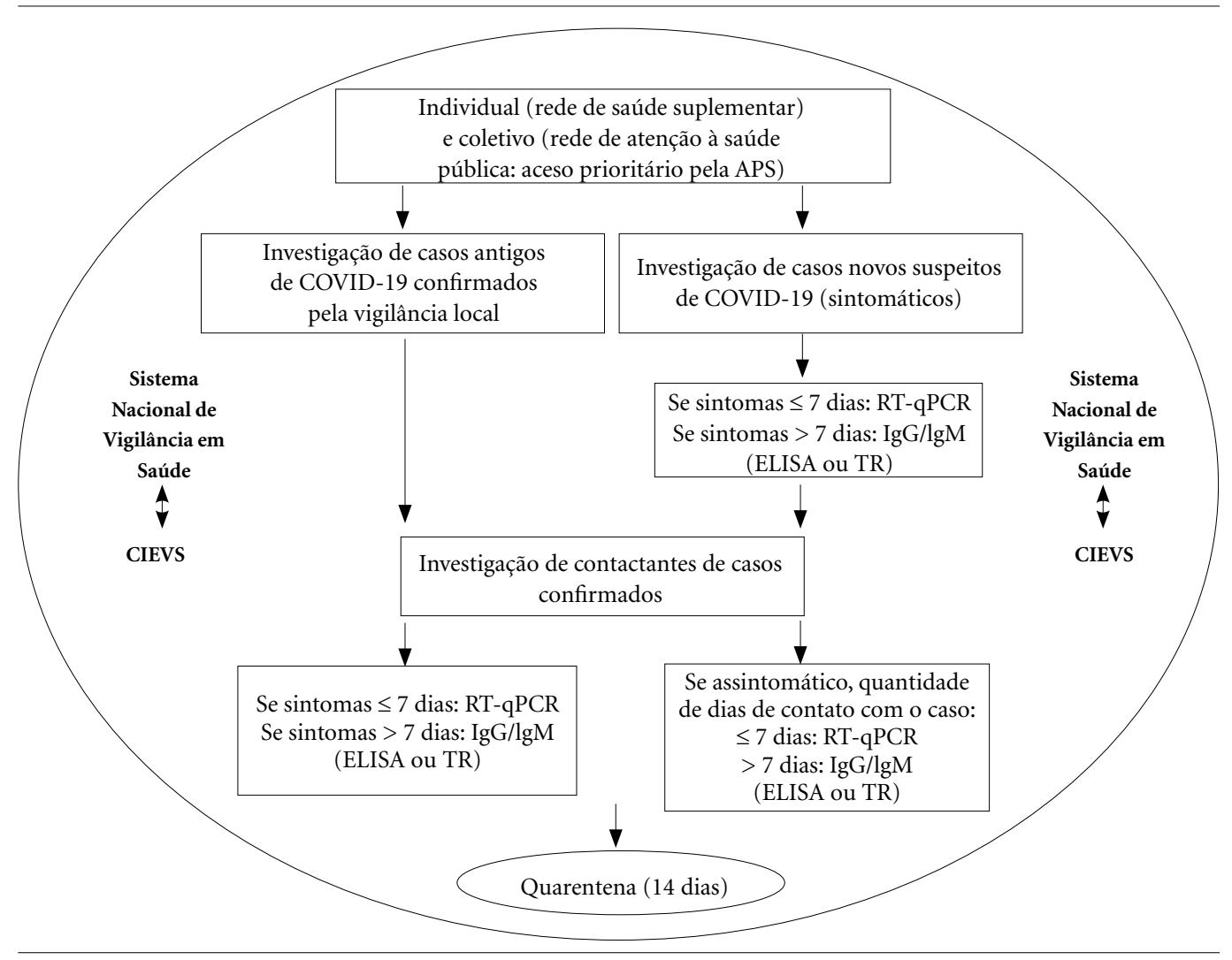

Figura 1. Resposta integrada de testagem para casos suspeitos de COVID-19 e seus contactantes. 
um processo de desfinanciamento crônico e ameaças constantes ao sistema público de saúde, dispomos de mecanismos gerenciais e de vigilância epidemiológica descentralizados capazes de uma resposta adequada ao desafio posto. Além disso, contamos com uma rede de laboratórios, universidades e institutos de pesquisa públicos em todos os estados da federação, que podem se constituir em uma rede de serviço diagnóstico, sob a coordenação do SNVS, para ampliação de testagem da
COVID-19. Para tanto, faz-se necessária a eliminação de impasses burocráticos para a acreditação dos laboratórios das Universidades, e de institutos de pesquisa para o diagnóstico; ampliação de financiamento para capacitação e contratação de pessoal; investimento em estudos de investigação acerca do diagnóstico sorológico, epidemiologia, desenvolvimento de vacinas e tratamento; articulação político-científica para tomada de decisão baseada em evidências científicas locais e globais.

\section{Colaboradores}

L Magno: concepção do artigo, revisão de literatura, redação e revisão final do artigo. TA Rossi, CC Santos, LM Marques, GB Campos, M Pereira e NMBL Prado: Redação, revisão de literatura e revisão final do artigo. FW Mendonça-Lima: Redação, revisão de literatura, revisão crítica do manuscrito, e revisão final do artigo. I Dourado: Redação, revisão de literatura, revisão crítica do manuscrito e aprovação final do artigo.

\section{Agradecimentos}

Agradecemos ao Movimento de Reforma Sanitária Brasileira por ter ajudado a fundar e erguer o Sistema Único de Saúde (SUS), constituindo-se como fonte de inspiração para todos nós sanitaristas brasileiros. Agradecemos também às universidades públicas brasileiras por ter nos formado e ser nosso espaço de trabalho. 


\section{Referências}

1. World Health Organization (WHO). Rolling updates on coronavirus disease (COVID-19). 2020. [acessado 2020 Maio 31]. Disponível em: https://www.who. int/emergencies/diseases/novel-13 coronavirus-2019/ events-as-they-happen

2. World Health Organization (WHO). Q $\triangleleft A$ on coronaviruses (COVID-19). 2020. [acessado 2020 Maio 31]. Disponível em: https://www.who.int/emergencies/ diseases/novel-coronavirus-2019/question-16 andanswers-hub/q-a-detail/q-a-coronaviruses

3. World Health Organization (WHO). COVID-19 Dashboard. 2020. [acessado 2020 Maio 12]. Disponível em: https://covid19.who.int/

4. Ferretti L, Wymant C, Kendall M, Zhao L, Nurtay A, Abeler-Dörner L, Parker M, Bonsall D, Fraser C. Quantifying SARS-CoV-2 transmission suggests epidemic control with digital contact tracing. Science 2020; 368(6491):eabb6936.

5. Huang L, Zhang X, Zhang X, Wei Z, Zhang L, Xu J, Liang P, Xu Y, Zhang C, Xu A. Rapid asymptomatic transmission of COVID-19 during the incubation period demonstrating strong infectivity in a cluster of youngsters aged 16-23 years outside Wuhan and characteristics of young patients with COVID-19: A prospective contact-tracing study. I Infect 2020; 80(6):e1-e13.

6. Wang Z, Tang K. Combating COVID-19: health equity matters. Nat Med 2020; 26(4):458.

7. Rodrigues NCP, Andrade MKN, O'Dwyer G, Flynn M, Braga JU, Almeida AS, Bastos LS, Lino VTS. Distribuição da tuberculose pulmonar no Rio de Janeiro (Brasil): Uma análise espacial. Cien Saude Colet 2017; 22(12):4125-4134.

8. Adepoju P. Tuberculosis and HIV responses threatened by COVID-19. Lancet HIV 2020; 7(5):e319-e320.

9. Zhou F, Yu T, Du R, Fan G, Liu Y, Liu Z, Xiang J, Wang Y, Song B, Gu X, Guan L, Wei Y, Li H, Wu X, Xu J, Tu S, Zhang Y, Chen H, Cao B. Clinical course and risk factors for mortality of adult inpatients with COVID-19 in Wuhan, China: a retrospective cohort study. Lancet 2020; 395(10229):1054-1062.

10. Chen N, Zhou M, Dong X, Qu J, Gong F, Han Y, Qiu Y, Wang J, Liu Y, Wei Y, Xia J, Yu T, Zhang X, Zhang L. Epidemiological and clinical characteristics of 99 cases of 2019 novel coronavirus pneumonia in Wuhan, China: a descriptive study. Lancet 2020; 395(10223):507-513.

11. Dietz W, Santos-Burgoa C. Obesity and its Implications for COVID-19 Mortality. Obesity 2020; 00:1-1.

12. World Bank (WB). GINI index (World Bank estimate) Brazil. 2020. [acessado 2020 Maio 12]. Disponível em: https://data.worldbank.org/indicator/SI.POV.GINI

13. Programa das Nações Unidas para o Desenvolvimento (PNUD). Relatório do Desenvolvimento Humano 2019: além do rendimento, além das médias, além do presente - desigualdades no desenvolvimento humano no século XXI. Nova York: PNUD; 2019.

14. Silveira D, Alvarenga D. Trabalho informal avança para $41,3 \%$ da população ocupada e atinge nível recorde, diz IBGE. G1. [acessado 2020 Maio 12]. Disponível em: https://g1.globo.com/economia/noticia/ 2019/08/30/trabalho-informal-avanca-para-413percent-da-populacao-ocupada-e-atinge-nivel-recorde -diz-ibge.ghtml
15. GBD 2016 Brazil Collaborators. Burden of disease in Brazil, 1990-2016: a systematic subnational analysis for the Global Burden of Disease Study 2016. Lancet 2018; 392(10149):760-775.

16. Brasil. Ministério da Saúde (MS). Covid-19: painel coronavírus. Brasília: MS; 2020. [acessado 2020 Maio 31]. Disponível em: https://covid.saude.gov.br/

17. Rossetto EV, Luna EJA. A descriptive study of pandemic influenza A(N1N1)PDM09 in Brazil, 2009 2010. Revista do Instituto de Medicina Tropical de São Paulo 2016; 58:78.

18. Bastos LS, Niquini RP, Lana RM, Villela DAM, Cruz OG, Coelho FC, Codeço CT, Gomes MFC. COVID-19 e hospitalizações por SRAG no Brasil: uma comparação até a $12^{\mathrm{a}}$ semana epidemiológica de 2020. Cad Saude Publica 2020; 36(4):e00070120.

19. Fundação Oswaldo Cruz (Fiocruz), Brasil. Ministério da Saúde (MS). Monitoramento de casos reportados de síndrome respiratória aguda grave (SRAG) hospitalizados. 2020. [acessado 2020 Maio 12]. Disponível em: http://info.gripe.fiocruz.br/

20. COVID-19 Brasil. COVID-19: análise subnotificação. 2020. [acessado 2020 Maio 12]. Disponível em: https://ciis.fmrp.usp.br/covid19/analise-subnotificacao/

21. Prado M, Bastos L, Batista A, Antunes B, Baião F, Maçaira P, Hamacher S, Bozza F. Análise de subnotificação do número de casos confirmados da COVID-19 no Brasil. Núcleo de Operações e Inteligência em Saúde (NOIS) 2 Metodologia. 2020. [acessado 2020 Maio 12]. Disponível em: https://www.who.int/docs/ default-source/coronaviruse/who-china-joint-mission-on-covid-19-final-

22. Hasell J. Testing data provides us with two indicators of the quality of data on COVID-19.2020. [acessado 2020 Maio 12]. Disponível em: https://ourworldindata.org/ what-can-data-on-testing-tell-us-35 about-the-pandemic

23. Okba NMA, Müller MA, Li W, Wang C, Geurtsvankessel CH, Corman VM, Lamers MM, Sikkema RS, Bruin E, Chandler FD, Yazdanpanah Y, Le Hingrat Q, Descamps D, Houhou-Fidouh N, Reusken CBEM, Bosch BJ, Drosten C, Koopmans MPG, Haagmans BL. SARSCoV-2 specific antibody responses in COVID-19 patients. Emerg Infect Dis 2020; 26(7):1478-1488.

24. Barifouse R. Coronavírus: por que o Brasil ainda não conseguiu fazer testes em massa? BBC News Brasil 2020. [acessado 2020 Maio 12]. Disponível em: https://www.bbc.com/portuguese/brasil-41 52145795

25. Onofre R. Sem insumos, laboratórios privados limitam exames para detectar coronavírus. Folha de São Paulo 2020. [acessado 2020 Maio 12]. Disponível em: https:// www1.folha.uol.com.br/equilibrioesaude/2020/03/ sem-insumos-45laboratorios-privados-limitam-exames- para-detectar-coronavirus.shtml 46

26. Loeffelholz MJ, Tang YW. Laboratory diagnosis of emerging human coronavirus infections-the state of the art. Emerg Microbes Infect 2020; 9(1):747-756.

27. Yeo C, Kaushal S, Yeo D. Enteric involvement of coronaviruses: is faecal-oral transmission of SARS-CoV-2 possible? Lancet Gastroenterol Hepatol 2020; 5(4):335337. 
28. Lou B, Li T-D, Zheng S-F, Su Y-Y, Li Z-Y, Liu W, Yu F, Ge SX, Zou QD, Yuan Q, Lin S, Hong CM, Yao XY, Zhang XJ, Wu DH, Zhou GL, Hou WH, Li TT, Zhang YL, Zhang SY, Fan J, Zhang J, Xia NS, Chen Y. Serology characteristics of SARS-CoV-2 infection since the exposure and post symptoms onset. Eur Respir J 2020; 2000763.

29. Pan Y, Zhang D, Yang P, Poon LLM, Wang Q. Viral load of SARS-CoV-2 in clinical samples. Lancet Infectious Diseases 2020; 20:411-412.

30. Negri F, Zucoloto G, Miranda P, Koeller P. Ciência e Tecnologia frente à pandemia: Como a pesquisa científica e a inovação estão ajudando a combater o novo coronavírus no Brasil e no mundo. Centro de Pesquisa em Ciência, Tecnologia e Sociedade 2020. [acessado 2020 Maio 12]. Disponível em: https:// www.ipea.gov.br/cts/pt/central-15 de-conteudo/artigos/artigos/182-corona

31. Guo L, Ren L, Yang S, Xiao M, Chang D, Yang F, Cruz CSD, Wang Y, Wu C, Xiao Y, Zhang L, Han L, Dang S, Xu Y, Yang Q, Xu S, Zhu H, Xu Y, Jin Q, Sharma L, Wang L, Wang J. Profiling Early Humoral Response to Diagnose Novel Coronavirus Disease (COVID-19). Clin Infect Dis 2020; ciaa310.

32. Petherick A. Developing antibody tests for SARSCoV-2. Lancet 2020; 395(10230):1101-1102.

33. Tang YW, Schmitz JE, Persing DH, Stratton CW. The Laboratory Diagnosis of COVID-19 Infection: Current Issues and Challenges. J Clin Microbiol 2020; 58(6):e00512-20.

34. Li Q, Guan X, Wu P, Wang X, Zhou L, Tong Y, Ren R, Leung KSM, Lau EHY, Wong JY, Xing X, Xiang N, Wu Y, Li C, Chen Q, Li D, Liu T, Zhao J, Liu M, Tu W, Chen C, Jin L, Yang R, Wang Q, Zhou S, Wang R, Liu H, Luo Y, Liu Y, Shao G, Li H, Tao Z, Yang Y, Deng Z, Liu B, Ma Z, Zhang Y, Shi G, Lam TTY, Wu JT, Gao GF, Cowling BJ, Yang B, Leung GM, Feng Z. Early transmission dynamics in Wuhan, China, of novel coronavirus-infected pneumonia. $N$ Engl J Med 2020; 382(13):1199-1207.

35. Reusken CBEM, Broberg EK, Haagmans B, Meijer A, Corman VM, Papa A, Charrel R, Drosten C, Koopmans M, Leitmeyer K, On Behalf Of Evd-LabNet And Erli-Net. Laboratory readiness and response for novel coronavirus (2019-nCoV) in expert laboratories in 30 EU/EEA countries, January 2020. Euro Surveill 2020; 25(6):2000082.

36. National Health Commission \& State Administration of Traditional Chinese Medicine. Diagnosis and Treatment Protocol for Novel Coronavirus Pneumonia. 2020. [acessado 2020 Maio 12]. Disponível em: http://www. kankyokansen.org/uploads/uploads/files/jsipc/protocol_V7.pdf 36

37. Jin Y, Yang H, Ji W, Wu W, Chen S, Zhang W, Duan G. Virology, epidemiology, pathogenesis, and control of covid-19. Viruses 2020; 12(4):372.

38. Uys PW, Warren R, van Helden PD, Murray M, Victor TC. Potential of rapid diagnosis for controlling drug-susceptible and drug-resistant tuberculosis in communities where Mycobacterium tuberculosis infections are highly prevalent. J Clin Microbiol 2009; 47(5):1484-1490.
39. Okeke IN, Manning RS, Pfeiffer T. Diagnostic schemes for reducing epidemic size of african viral hemorrhagic fever outbreaks. J Infect Dev Ctries 2014; 8(9):1148-1159.

40. Rong X, Yang L, Chu H, Fan M. Effect of delay in diagnosis on transmission of COVID-19. Mathematical Biosciences and Engineering 2020; 17:2725-2740.

41. Ng Y, Li Z, Chua YX, Liang W, Zhao Z, Er B, Pung R, Chiew CJ, Lye DC, Heng D, Lee VJ. Morbidity and Mortality Weekly Report Evaluation of the Effectiveness of Surveillance and Containment Measures for the First 100 Patients with COVID-19 in Singapore MMWR Morb Mortal Wkly Rep 2020; 69(11):307-311

42. Peto J. Covid-19 mass testing facilities could end the epidemic rapidly. BMJ 2020; 368:m1163.

43. Beeching NJ, Fletcher TE, Beadsworth MBJ. Covid-19: Testing times. BMJ 2020; 369:m1403.

44. Brasil. Ministério da Saúde (MS). Emergência de Saúde Pública de Importância Nacional pela Doença pelo Coronavírus 2019: Vigilância Integrada de Síndromes Respiratórias Agudas Doença pelo Coronavírus 2019, Influenza e outros vírus respiratórios. Brasília: MS; 2020. [acessado 2020 Maio 12]. Disponível em: https://portalarquivos.saude.gov.br/images/pdf/2020/ April/07/GuiaDeVigiEpidemC19-v2.pdf

45. Legido-Quigley H, Asgari N, Teo YY, Leung GM, Oshitani H, Fukuda K, Cook AR, Hsu LY, Shibuya K, Heymann D. Are high-performing health systems resilient against the COVID-19 epidemic? Lancet 2020; 395:848-850.

46. Cousins S. New Zealand eliminates COVID-19. Lancet 2020; 395:1474.

47. New Zealand's elimination COVID-10. [acessado 2020 Maio 12]. Disponível em: https://www.nzma.org.nz/ journal-articles/new-zealands-elimination-strategyfor-the-covid-19-pandemic-and-what-is-requiredto-make-it-work

48. Australian Government. Department of Health. Coronavirus (COVID-19) current situation and case numbers. 2020. [acessado 2020 Maio 12]. Disponível em: https://www.health.gov.au/news/health-alerts/ novel-coronavirus-2019-ncov-health-alert/coronavirus-covid-19-current-situation-and-case-numbers

49. Paterlini M. On the front lines of coronavirus: The Italian response to covid-19. BMJ 2020; 368:m1065.

50. Tanne JH, Hayasaki E, Zastrow M, Pulla P, Smith P, Rada AG. Covid-19: How doctors and healthcare systems are tackling coronavirus worldwide. BMJ 2020; 368:m1090.

51. Lacobucci G. Covid-19: What is the UK's testing strategy? BMJ (Clinical research ed) 2020; 368:m1222.

52. de Lusignan S, Lopez Bernal J, Zambon M, Akinyemi $\mathrm{O}$, Amirthalingam G, Andrews N, Borrow R, Byford R, Charlett A, Dabrera G, Ellis J, Elliot AJ, Feher M, Ferreira F, Krajenbrink E, Leach J, Linley E, Liyanage H, Okusi C, Ramsay M, Smith G, Sherlock J, Thomas N, Tripathy M, Williams J, Howsam G, Joy M, Hobbs R. Emergence of a Novel Coronavirus (COVID-19): Protocol for Extending Surveillance Used by the Royal College of General Practitioners Research and Surveillance Centre and Public Health England. JMIR Public Health and Surveillance 2020; 6(2):e18606. 
53. Stafford N. Covid-19: Why Germany's case fatality rate seems so low. BMJ 2020; 369:1-2.

54. Centers for Disease Control and Prevention (CDC). Cases in the U.S. 2020. [acessado 2020 Maio 12]. Disponível em: https://www.cdc.gov/coronavirus/ 2019ncov/cases-updates/cases-in-us.html

55. Lancet. COVID-19 in Brazil: "So what?" Lancet 2020; 395:1461.

56. Paim J, Travassos C, Almeida C, Bahia L, MacInko J. The Brazilian health system: History, advances, and challenges. Lancet 2011; 377(9779):1778-1797.

57. Hasell J, Ortiz-Ospina E, Mathieu E, Ritchie H, Beltekian DMB, Roser M. To understand the global pandemic, we need globas testing - the Our World in Data COVID-19 Testing dataset. Oxford: University of Oxford; 2020.

58. Teixeira MG, Costa MCN, Carmo EH, Oliveira WK, Penna GO. Health surveillance at the SUS: Development, effects and perspectives. Cien Saude Colet 2018; 23(6):1811-1818.

59. Brasil. Ministério da Saúde (MS). Centro de informações estratégicas em vigilância em saúde-CIEVS. Brasília: MS; 2020. [acessado 2020 Maio 12]. Disponível em: https://www.saude.gov.br/vigilancia- emsaude/emergencia-em-saude-publica/cievs

60. Nascimento L. Anvisa aprova testes rápidos para covid-19 em farmácias. 2020. [acessado 2020 Maio 12]. Disponível em: https://agenciabrasil.ebc.com. br/saude/noticia/2020-04/anvisa-aprova-testes-rapidos-para-covid-19-em-farmacias

61. Agência Nacional de Vigilância Sanitária (Anvisa). Covid-19: aprovados seis novos testes. 2020. [acessado 2020 Maio 12]. Disponível em: http://portal. anvisa.gov.br/noticias/-/asset_publisher/FXrpx9qY7FbU/content/covid-19-aprovados-seis-novos-testes/219201

62. Agência Nacional de Vigilância Sanitária (Anvisa). Aprovados primeiros testes rápidos para Covid-19. 2020. [acessado 2020 Maio 12]. Disponível em: http:// portal.anvisa.gov.br/noticias/-/asset_publisher/ FXrpx9qY7FbU/content/aprovados-primeiros-testes-rapidos-para-covid-19/219201

63. Brasil. Ministério da Saúde (MS). Programa Diagnosticar para Cuidar prevê ações de testagem em 2020. 2020. [acessado 2020 Maio 12]. Disponível em: https:// www.saude.gov.br/noticias/agencia-saude/46848programa-diagnosticar-para-cuidar-preve-acoes-detestagem-em-2020

64. Brasil. Ministério da Saúde (MS). Acurácia dos diagnósticos registrados para COVID-19. Brasília: MS; 2020. [acessado 2020 Maio 12]. Disponível em: https://portalarquivos.saude.gov.br/images/pdf/2020/ April/29/Acuracia-Diagnosticos-COVID19.pdf
65. Castro R, Luz PM, Wakimoto MD, Veloso VG, Grinsztejn B, Perazzo H. COVID-19: a meta-analysis of diagnostic test accuracy of commercial assays registered in Brazil. Braz J Infect Dis 2020; 24(2):180-187.

66. Nadanovsky P. A utilidade do teste para detecção de anticorpos do SARS-CoV-2. Notícias UERJ 2020. [acessado 2020 Maio 12]. Disponível em: https:// www.ims.uerj.br/2020/04/27/a-utilidade-do-testepara-deteccao-de-anticorpos-do-sars-cov-2/

67. Estem Kristecia S, Catania J, Klausner JD. HIV Self-Testing: a Review of Current Implementation and Fidelity. Current HIV/AIDS Reports 2016; 13(2):107-115.

68. Brasil. Ministério da Saúde (MS). The HIV self-test in SUS. 2020. [acessado 2020 Maio 12]. Disponível em: http://www.aids.gov.br/pt-br/autoteste/o-autoteste-de-hiv-no-sus

69. World Health Organization (WHO), UNITAID. Landscape for HIV rapid diagnostic tests for HIV self-testing. 2015. [acessado 2020 Maio 12]. Disponível em: https://www.who.int/hiv/pub/vct/hiv-self-testing landscape_2015-unitaid-who.pdf?ua $=1$

70. Garnelo L, Lima JG, Rocha ESC, Herkrath FJ. Acesso e cobertura da Atenção Primária à Saúde para populações rurais e urbanas na região norte do Brasil. Saúde em Debate 2018; 42(n. esp. 1):81-99.

71. Nunes CA, Aquino R, Medina MG, Vilasbôas ALQ, Pinto Júnior EP, Luz LA. Visitas domiciliares no Brasil: características da atividade basilar dos Agentes Comunitários de Saúde. Saúde em Debate 2018; 42(n. esp. 2):127-144.

72. Brasil. Ministério da Saúde (MS). Testes Rápidos. 2020. [acessado 2020 Maio 12]. Disponível em: http://www. aids.gov.br/pt-br/profissionais-de-saude/testes-rapidos

Artigo apresentado em 01/06/2020

Aprovado em 01/06/2020

Versão final apresentada em 03/06/2020 